Since the factors in the first two sets of brackets are finite Blaschke products and the zero in the third is a convex combination of such, and since the coefficients are nonnegative and sum to 1 , the proof is complete.

\title{
REFERENCES
}

1. C. Caratheodory, Theory of functions. Vol. 2, Chelsea, New York, 1954.

2. R. R. Phelps, Extreme points in functions algebras, Duke Math. J. 32 (1965), 267-278.

Massachusetts Institute of Technology

\section{EXACTNESS OF INVERSE LIMITS}

\section{BY ULRICH OBERST}

Communicated by Saunders Mac Lane, May 20, 1968

I. The problem of this investigation is to characterize those small categories $X$ for which the inverse limit

$$
\operatorname{proj} \lim _{X}: A B^{X} \rightarrow A B
$$

is exact. Here $A B$ is the category of abelian groups, and $A B^{\boldsymbol{x}}$ is the category of functors from $X$ to $A B$. In this context I conjecture the following

TheOREM I. Let $X$ be a small category. Then the following assertions are equivalent:

(1) The inverse limit proj $\lim _{X}: A B^{\mathbf{x}} \rightarrow A B$ is exact.

(2) For every abelian category I with exact direct products, the inver se limit $\operatorname{proj} \lim _{X}: \mathfrak{A}^{\mathbb{X}} \rightarrow \mathfrak{A}$ is exact.

(3) Every connected component $Y$ of $X$ contains an object $y$ together with an endomorphism $e \in Y(y, y)$ such that the following properties are satisfied:

(i) $y$ is a smallest object of $Y$, i.e., for any object $z \in Y$ there is a morphism $y \rightarrow z$.

(ii) $e$ equalizes any two morphisms with the same codomain and domain $y$, i.e., any diagram $y \rightarrow \dot{\rightarrow} \mathrm{y}_{\rightarrow} z$ is commutative.

At present, I can prove the equivalence of (1) and (2) and the implication (3) $\rightarrow(1)$ in general, i.e., without any additional condition on $X$. The implication $(1) \rightarrow(3)$ holds at least if one of the following conditions on $X$ is satisfied: 
(a) $X$ is a monoid, i.e., has exactly one object. In this case (3) means that there is a right zero element $e \in X$ with $\alpha e=e$ for all $\alpha \in X$.

(b) $X$ satisfies the condition

$\left(\mathrm{F} 2^{\prime}\right)^{\circ}$ : Every commutative diagram $x_{1} \rightarrow x_{2} \rightarrow x_{3}$ can be extended to a commutative diagram $x_{0} \rightarrow x_{1} \rightarrow x_{2} \rightarrow x_{3}$.

This condition is satisfied in particular if the connected components $Y$ of $X$ are filtered from below (see also $[1$, p. 7$]$ ), i.e., if $X$ satisfies the condition

$(\mathrm{F} 2)^{\circ}$ : Any diagram $x_{1} \rightrightarrows x_{2}$ in $X$ can be completed to a commutative diagram $x_{0} \rightarrow x_{1} \rightarrow x_{2}$.

II. The following steps are taken in the proof of Theorem I.

Step 1 . It is clear that for any abelian category $\mathfrak{A}$ with exact direct products the inverse limit proj $\lim _{X}: \mathfrak{O}^{\boldsymbol{X}} \rightarrow \mathfrak{U}$ is exact if and only if for every connected component $Y$ of $X$ the inverse limit proj $\lim _{Y}: \mathfrak{A}^{Y} \rightarrow \mathfrak{U}$ is exact. Hence one may assume without loss of generality that $X$ itself is connected.

Step 2. Let $\boldsymbol{Z}$ denote the group of integers, and let $8: X \rightarrow A B$ be the constant functor with values $\boldsymbol{Z}$. Let

$$
\operatorname{Hom}_{X}:\left(A B^{\boldsymbol{X}}\right)^{0} \times \mathfrak{A}^{\boldsymbol{x}} \rightarrow \mathfrak{U}
$$

be the formal Hom-functor, defined for any abelian category $\mathfrak{A}$ with direct products (see e.g. $[4$, p. $145 \mathrm{ff}]$ ). In the same fashion let

$$
\underset{X^{0}}{\otimes}: A B^{x} \times \mathfrak{U}^{x^{0}} \rightarrow \mathfrak{U}
$$

be the tensor product, defined for any abelian category $\mathfrak{A}$ with direct sums (loc. cit.). It is easily seen that

$$
\operatorname{proj} \lim _{x}=\operatorname{Hom}_{X}(\mathfrak{B}, ?): \mathfrak{P}^{x} \rightarrow \mathfrak{A}
$$

respectively

$$
\text { inj } \lim _{X^{0}}=\mathfrak{Z} \underset{X^{0}}{\otimes} ?: \mathfrak{U}^{X^{0}} \rightarrow \mathfrak{U}
$$

where $X^{0}$ is the dual category of $X$.

Using these notions we prove the

Proposition. Let $X$ be a small category. Then the following properties are equivalent.

(1) proj $\lim _{X}: A B^{x} \rightarrow A B$ is exact.

(2) For any abelian category $\mathfrak{O}$ with exact direct products the functor proj $\lim _{X}: \mathfrak{U}^{\boldsymbol{X}} \rightarrow \mathfrak{U}$ is exact.

(3) The object $B \in A B^{X}$ is projective. 
Moreover, if (1)-(3) are satisfied then for any abelian category $\mathfrak{A}$ with exact direct sums the direct limit

$$
\text { inj } \lim _{X^{0}}: \mathfrak{X}^{X^{0}} \rightarrow \mathfrak{A}
$$

is exact.

The basic observation for this proof is that $B$ is projective if and only if it is $X$-representable in the sense of Eilenberg-Mac Lane [2], i.e., if and only if the canonical epimorphism

$$
\begin{aligned}
& \phi: \prod_{x \in X} Z X(x, ?) \rightarrow Z, \\
& i d_{x} 1 \in 3 x=Z
\end{aligned}
$$

splits. Here $\boldsymbol{Z} X(x, y)$ is the free abelian group with basis $X(x, y)$.

The preceding proposition can be generalized to the

Proposition. For a small category $X$ and a nonnegative integer $n$ the following assertions are equivalent:

(1) The $(n+1)$ st right derived functor $\left(\text { proj } \lim _{X}\right)_{n+1}$ of proj $\lim _{X}$ : $A B^{x} \rightarrow A B$ is zero.

(2) The $(n+1)$ st right derived functor $\left(\text { proj } \lim _{X}\right)_{n+1}$ of proj $\lim _{X}: \mathfrak{A}^{X}$ $\rightarrow \mathfrak{U}$ is zero for any abelian category $\mathfrak{A}$ with exact direct products.

(3) The projective dimension of $\boldsymbol{Z}$ is smaller than or equal to $n$.

Step 3. The most important step in proving Theorem I is the following proposition which is a special case of the implication $(1) \rightrightarrows(3)$ of Theorem I.

Proposition. Let $X$ be a small, connected category. Assume that proj $\lim _{X}: A B^{x} \rightarrow A B$ is exact, and that $X$ is filtered from below, i.e., satisfies (F2) ${ }^{\circ}$. Then there are an object $x \in X$ and an endomorphism $e \in X(x, x)$ satisfying the conditions (i) and (ii) of (3), Theorem I.

The proof of this proposition starts with the fact that the canonical isomorphism

$$
\phi: \prod_{x \in X} z X(x, ?) \rightarrow 3
$$

splits.

Step 4. Under the hypotheses (a), respectively (b), on $X$ the implication $(1) \rightarrow(3)$ of Theorem I (assume $X$ connected without loss of generality) is proven in the following way: assume that proj $\lim _{X}: A B^{x}$ $\rightarrow A B$ is exact. From the proposition of the second step we conclude that inj $\lim _{X^{0}}: A B^{x^{0}} \rightarrow A B$ is exact. Moreover $X^{0}$ satisfies the dual conditions (a) ${ }^{0}$ respectively (b) ${ }^{0}$ of (a) respectively (b). Under these 
hypotheses it was shown in [3] respectively [5] that $X^{0}$ is filtered from above. Hence $X$ is filtered from below. Using this fact and the proposition of the third step we obtain that $X$ satisfies the condition (3) of Theorem I.

Step 5. In order to prove Theorem I in general it is sufficient to prove the following

ConJeCture: Let $X$ be a connected, small category and assume that inj $\lim _{X}: A B^{x} \rightarrow A B$ is exact. Then $X$ is filtered from above.

I conjecture this result because it is true if $X$ is a monoid [3] or satisfies $\left(\mathrm{F} 2^{\prime}\right)[5]$, and no counterexamples are known.

\section{REFERENCES}

1. M. Artin, Grothendieck topologies, Seminar Notes, Harvard University 1962.

2. S. Eilenberg and S. Mac Lane, Acyclic models, Amer. J. Math. 75 (1953), 189199.

3. J. R. Isbell, (to appear).

4. B. Mitchell, Theory of categories, Academic Press, New York, 1965.

5. U. Oberst, Homology of categories and exactness of direct limits, Math. Z. (to appear.)

University of Chicago 